\title{
On Bifurcation from Infinity and Multipoint Boundary Value Problems
}

\author{
Guy Degla ${ }^{1,2}$ \\ ${ }^{1}$ African University of Science and Technology (AUST), Abuja, Nigeria \\ ${ }^{2}$ Institut de Mathematiques et de Sciences Physiques (IMSP), Porto-Novo, Benin \\ Email: gadegla@yahoo.fr \\ Received 12 January 2014; revised 12 February 2014; accepted 18 February 2014 \\ Copyright (C 2014 by author and Scientific Research Publishing Inc. \\ This work is licensed under the Creative Commons Attribution International License (CC BY). \\ http://creativecommons.org/licenses/by/4.0/ \\ (c) (1) Open Access
}

\begin{abstract}
We generalize a result on bifurcation from infinity of high order ordinary differential equations with multi-point boundary conditions. Our abstract setting represents a variant of Nonlinear Krein-Ruthman theorems. Furthermore, an analysis of this abstract setting raises an open question motivated by some misunderstanding and inconclusive proofs about the simplicity of principal eigenvalues in some articles in the literature.
\end{abstract}

\section{Keywords}

Krein-Rutman, Positivity, Cone, Homogeneity, Compactness, Eigenvalue, Simplicity, Bifurcation

\section{Introduction}

In this paper, we generalize and improve a result of Coyle et al. [1] about the bifurcation from infinity after stating in the line of Nussbaum [2], Schmitt [3], etc., a type of nonlinear Krein-Rutman theorem for a class of positively 1-homogeneous, compact and continuous operators in Banach spaces leaving invariant cones.

Our method is motivated by the maximum principle of Degla [4] and a result on the principal eigenvalue of multi-point Boundary Value Problems (BVP's) of Degla [5] which allow the use of cone theoretic arguments and of the well-known general result on bifurcation from infinity; see Coyle [1], Mawhin [6] and Rabinowitz [7].

Furthermore, in our abstract setting, the nonlinear Krein-Rutman Theorem resets an important result on the simplicity of positive eigenvalues [8] by avoiding some inconclusive argument [8] (page 3086, lines 29-37) also misused in [9] (page 550, lines 15-27). However the gap in their arguments under their assumptions, remains an open question. 


\section{Preliminary Definitions and Notations}

We say that a nonempty subset $\mathcal{K}$ of a Banach space $X$ is a cone if it is closed and

1) $\mathcal{K}+\mathcal{K} \subset \mathcal{K}$

2) $\lambda \mathcal{K} \subset \mathcal{K}, \forall \lambda \geq 0$ and

3) $\mathcal{K} \cap(-\mathcal{K})=\{0\}$.

In other words, the cones considered here are closed convex cones with vertex at 0 .

A cone $\mathcal{K}$ of a Banach space $X$ induces a partial ordering on $X$ by the relation

$$
x \preceq y \text { if and only if } y-x \in \mathcal{K} \text {, }
$$

and it follows that

$$
x \succeq 0 \text { if and only if } x \in \mathcal{K} \text {. }
$$

Therefore $(X, \mathcal{K})$ is called an ordered Banach space with $\mathcal{K}$ as the positive cone of $X$. Note that we write $x \prec y$ when $x \preceq y$ and $x \neq y$; i.e.,

$$
x \prec y \text { if and only if } y-x \in \mathcal{K} \backslash\{0\} .
$$

A cone $\mathcal{K}$ of a Banach space $X$ is said to be generating if $X=\mathcal{K}-\mathcal{K}$, and total if $X=\overline{\mathcal{K}}-\mathcal{K}$.

Given a Banach space $X$ with dual $X^{\prime}$, if a cone $\mathcal{K}$ of $X$ is generating, then the set defined by

$$
\mathcal{K}^{\prime}:=\left\{f \in X^{\prime} /\langle f, u\rangle \geq 0, \forall u \in \mathcal{K}\right\}
$$

is a cone of $X^{\prime}$ called the dual cone of $\mathcal{K}$.

The positive cone $\mathcal{K}$ of an ordered Banach space $X$ is said to be normal if there exists a positive constant $c \geq 1$ such that

$$
\forall x, y \in \mathcal{K}, \quad x \preceq y \Rightarrow\|x\| \leq c\|y\| .
$$

When $c=1$, such an ordered Banach space is said to be monotone.

Let $(X, \mathcal{K})$ be an ordered Banach space. Then

- A linear operator $T: X \rightarrow X$ is said to be positive if

$$
T(\mathcal{K}) \subset \mathcal{K},
$$

and strongly positive if

$$
T(\mathcal{K} \backslash\{0\}) \subset \dot{\mathcal{K}}
$$

- An arbitrary operator $T: X \rightarrow X$ is said to be increasing if

$$
\forall x, y \in X, \quad x \preceq y \Rightarrow T x \preceq T y,
$$

strictly increasing if

$$
\forall x, y \in X, \quad x \prec y \Rightarrow T x \prec T y,
$$

and strongly increasing if

$$
\forall x, y \in X, \quad x \prec y \Rightarrow T y-T x \in \stackrel{\mathcal{K}}{ } .
$$

We shall say that $T$ is increasing on $\mathcal{K}$ if

$$
\forall x, y \in \mathcal{K}, \quad x \preceq y \Rightarrow T x \preceq T y .
$$

Observe that if an operator $T$ is increasing on $\mathcal{K}$ and satisfies $T(0)=0$, then it leaves invariant $\mathcal{K}$.

Besides in our applications, we shall use the following terminology based on Degla [4] [5], Elias [10] and Coppel [11]. Given fixed positive integers $n, m$ and $k_{1}, \cdots, k_{m}$ such that $2 \leq m \leq \sum_{i=1}^{m} k_{i}=n$, and real numbers $a=a_{1}<\cdots<a_{m}=b$, we shall denote by $P$ the Levin's polynomial defined by $P(t)=\prod_{i=1}^{m}\left(t-a_{i}\right)^{k_{i}}$ and we shall deal with disconjugate $n^{\text {th }}$ order differential operators on $[a, b]$ of the form 


$$
L x:=x^{(n)}+p_{1}(t) x^{(n-1)}+\cdots+p_{n}(t) x
$$

where the coefficients $p_{1}, \cdots, p_{n}$ are given continuous functions on $[a, b]$, that is, an $n^{\text {th }}$-order differential linear operator $L$ such that every nontrivial solution of the differential equation $L x=0$ has less than $n$ zeros counting their multiplicities.

Recall that an $n^{\text {th }}$-order differential linear operator $L$;

$$
L x \equiv x^{(n)}+p_{1}(t) x^{(n-1)}+\cdots+p_{n}(t) x
$$

with $p_{1}, \cdots, p_{n} \in \mathcal{C}([a, b])$, is disconjugate on $[a, b]$ if and only if $L$ has a Polya factorization; that is, there exist $n$ smooth positive functions $v_{i} \in \mathcal{C}^{n-i+1}([a, b]), 1 \leq i \leq n$, such that

$$
\begin{gathered}
L x=v_{1} \cdots v_{n} D \frac{1}{v_{n}} D \cdots D \frac{1}{v_{1}} x \text { for every } x \in \mathcal{C}^{n}([a, b]) ; \\
\text { where } D=\frac{\mathrm{d}}{\mathrm{d} t}
\end{gathered}
$$

cf. [11].

Furthermore $G$ will denote the Green function associated to the Boundary Value Problems (in short BVP's)

$$
\left\{\begin{array}{l}
L x=0, \\
x^{(j)}\left(a_{i}\right)=0,1 \leq i \leq m, 0 \leq j \leq k_{i}-1,
\end{array}\right.
$$

and besides, given $f \in \mathcal{C}([a, b])$, we shall adopt the notation $\|f\|_{\infty}=\sup _{a \leq t \leq b}|f(t)|$ and

$$
S_{f}(t)=\left\{\begin{array}{ll}
\frac{f(t)}{|f(t)|} & \text { if } f(t) \neq 0 \\
0 & \text { if } f(t)=0
\end{array} .\right.
$$

As in [5], we shall also consider the Banach space

$$
X=\{u \in \mathcal{C}([a, b]):|u(t)| \leq c|P(t)|, a \leq t \leq b ; \text { for a positive constant } c=c(u)\}
$$

equipped with the norm

$$
\|u\|_{P}=\|u\|_{\infty}+\sup _{t \neq a_{1}, \cdots, a_{m}}\left|\frac{u(t)}{P(t)}\right|
$$

and ordered by the cone

$$
\mathcal{K}=\left\{u \in X: S_{P}(t) u(t) \geq 0, a \leq t \leq b\right\} .
$$

Now we are ready to state a variant of nonlinear Krein-Rutman theorems.

Proposition 1.1. Let $X$ be a real Banach space, $\mathcal{K}$ a nontrivial cone in $X$ and assume that $T: X \rightarrow X$ is a positively 1-homogeneous, compact and continuous operator.

a) If $T$ is increasing on $\mathcal{K}$ and there exist a positive vector $u \succ 0$, a positive real number $\omega$ and $a$ positive integer $m$, such that

\[ \omega T^{m} u \succeq u \text {, } \]
then $T$ has a positive eigenvalue $\lambda_{o} \geq \omega^{-\frac{1}{m}}$ with a positive eigenvector.

In case that $T$ is linear, its spectral radius $r(T)$ is such a positive eigenvalue and satisfies

$$
r(T)=\max \left\{\alpha^{-\frac{1}{m}} ; \alpha>0, \exists v \succ 0, \alpha T^{m} v \succeq v\right\} .
$$

b) If $\mathcal{K}$ has a nonempty interior $\mathcal{K}$ and $T \neq \equiv$ with the property

$$
\forall x_{1}, x_{2} \in \mathcal{K}, \quad x_{2}-x_{1} \in \mathcal{K} \Rightarrow T x_{2}-T x_{1} \in \mathcal{K} \cup\{0\},
$$


then $T$ has a unique positive eigenvalue and a unique positive normalized eigenvector.

In case that $T$ is linear, this positive eigenvalue coincides with the spectral radius $r(T)$ of $T$, is algebraically simple and has the following variational characterization:

$$
r(T)=\max \left\{\frac{1}{\alpha} ; \alpha>0, \exists v \succ 0, \alpha T v \succeq v\right\} .
$$

Remark 1.1. For a linear operator $T$, the condition (ii) of b) is equivalent to

$$
\forall x \in X, \quad x \in \mathcal{K} \Rightarrow T x \in \mathcal{K} \cup\{0\} .
$$

Furthermore the conclusion of b) can be heuristically motivated by the application of the Krein-Rutman theorem to the quotient space $X / \operatorname{ker} T$.

Remark 1.2. The above theorem is readily applicable to any positively 1 -homogeneous, compact and continuous operators that are strongly positive on the cone of an ordered Banach space.

Remark 1.3. The proof of Theorem 2 of [8] does not fully hold but is valid for strongly increasing operators. The reason is that its conclusion (2.9) is not correct and should be read $\underline{\lambda_{0} \leq \lambda}$ which does not contradict the inequality (2.10) therein; that is $\lambda_{0} \geq \lambda$.

The fact is that for instance in the Banach space $\mathbb{R}^{2}$ ordered by the cone

$$
K=\mathbb{R}_{+}^{2}=\left\{\left(x_{1}, x_{2}\right): x_{1} \geq 0, x_{2} \geq 0\right\}
$$

we have

$$
u:=(2,2) \succ v:=(1,2)
$$

and so with $\alpha=\beta=1>0$ it is clear that

$$
\alpha u \succ \beta v \nRightarrow \alpha>\beta .
$$

Likewise the inequality " $x_{0} \succ-\delta_{x_{0}}\left(x_{1}\right) x_{1}$ ” of the paragraph 4 of the proof of theorem 4.8 of [9] does not contradict the definition of " $\delta_{x_{0}}\left(x_{1}\right) x_{1}$ ” as can be seen with $x_{0}:=(2,2)$ and $x_{1}:=-(1,2)$ for which $\delta_{x_{0}}\left(x_{1}\right)=1$ by simply considering again the ordered Banach space $\left(\mathbb{R}^{2}, \mathbb{R}_{+}^{2}\right)$.

Therefore we are led to raise the following

Open Question: Does there exist a strictly increasing and positively 1-homogeneous compact operator of which positive eigenvalue is not simple?

Remark 1.4. For a positive compact linear operator $T$, the condition (i) of Part a) of Proposition 1.1 is equivalent to

$$
r(T)>0 \text {. }
$$

The following example illustrates Proposition 1.1.

Example 1.5. Consider the system of boundary value problems:

$$
\left\{\begin{array}{l}
-x^{\prime \prime}=\lambda Q(t) x, \\
x(0)=x(1)=0,
\end{array}\right.
$$

with $\lambda$ as a real parameter and

$$
Q=\left(\begin{array}{ll}
q_{1,1} & q_{1,2} \\
q_{2,1} & q_{2,2}
\end{array}\right)
$$

where the $q_{i, j}$ are assumed to be nonnegative continuous functions on [0,1] such that on the one hand $q_{1,1}$ and $q_{2,1}$ have a common support $\mathcal{S}_{1}$, and on the other hand $q_{1,2}$ and $q_{2,2}$ have a common support $\mathcal{S}_{2}$ such that $\mathcal{S}_{1} \cup \mathcal{S}_{2} \neq \varnothing$; i.e. $Q \neq \equiv$, and where the unknown vector-valued function $x=\left(\begin{array}{c}x_{1} \\ x_{2}\end{array}\right)$ is clearly searched in $\mathcal{C}^{2}\left([0,1] ; \mathbb{R}^{2}\right)$ with zero Dirichlet boundary condition. 
Then this system has a unique normalized solution with positive component functions on the interval $(0,1)$ corresponding to a unique positive value of the parameter $\lambda$.

Justification. We shall make use of Proposition 1.1 for the sake of illustration that may motivate other interesting works. Indeed it is immediately seen that for nontrivial solutions, we have $\lambda \neq 0$, and the system of BVPs

$$
\left\{\begin{array}{l}
-x^{\prime \prime}=\lambda Q x \\
x(0)=x(1)=0
\end{array}\right.
$$

is equivalent to the integral equation

$$
\int_{0}^{1} g(t, s) Q(s) x(s) \mathrm{d} s=\frac{1}{\lambda} x(t), \quad 0 \leq t \leq 1 ;
$$

with

$$
g(t, s)= \begin{cases}t(1-s), & 0 \leq t \leq s \leq 1 \\ s(1-t), & 0 \leq s \leq t \leq 1\end{cases}
$$

Moreover by considering the special space of continuous vector-valued functions

$$
X=\left\{u \in \mathcal{C}\left([0,1], \mathbb{R}^{2}\right): \frac{u(t)}{t(t-1)} \text { is bounded on the interval }(0,1)\right\}
$$

endowed with the norm $\|\cdot\|_{P}$ defined for any $u=\left(u_{1}, u_{2}\right) \in X$ by

$$
\|u\|_{P}=\sum_{i=1}^{2}\left(\left\|u_{i}\right\|_{\infty}+\sup _{0<t<1}\left|\frac{u_{i}(t)}{t(t-1)}\right|\right),
$$

which contains all possible solutions of our eigenvalue problem, and by letting

$$
\mathcal{K}=\left\{u=\left(\begin{array}{l}
u_{1} \\
u_{2}
\end{array}\right) \in X: u_{i} \geq 0 ; i=1,2\right\},
$$

we see that $(X, \mathcal{K})$ is a normal ordered Banach space. Furthermore the non-zero linear operator $T: X \rightarrow X$; $u \mapsto T u$ defined by

$$
T u(t)=\int_{0}^{1} g(t, s) Q(s) u(s) \mathrm{d} s, 0 \leq t \leq 1,
$$

is compact and satisfies

$$
T(\mathcal{K}) \subset \mathcal{K} \cup\{0\}
$$

with

$$
\mathcal{K}=\left\{u=\left(u_{1}, u_{2}\right) \in X: \inf _{0<t<1} \frac{u_{i}(t)}{t(1-t)}>0 ; i=1,2\right\},
$$

by the strong classical maximum principle.

The conclusion follows.

\section{Bifurcation from Infinity of Conjugate Multipoint BVPs}

This part can be considered as a more elaborated application of the main result of the previous section.

In the sequel we shall make use of the notations mentioned in Section 2. According to this,

$$
X=\{u \in \mathcal{C}([a, b]):|u(t)| \leq c|P(t)|, a \leq t \leq b ; \text { for a positive constant } c=c(u)\}
$$

equipped with the norm 


$$
\|u\|_{P}:=\|u\|_{\infty}+\sup _{t \neq a_{1}, \cdots, a_{m}}\left|\frac{u(t)}{P(t)}\right|
$$

and ordered by the cone

$$
\mathcal{K}=\left\{u \in X: S_{P}(t) u(t) \geq 0, a \leq t \leq b\right\}
$$

is an ordered Banach space.

Then the following theorem holds.

Theorem 2.1. Let $q \in \mathcal{C}([a, b])$ satisfy

$$
q \not \equiv 0 \text { and } S_{P}(t) q(t) \geq 0, \forall t \in[a, b] .
$$

Moreover let $h:[a, b] \times \mathbb{R} \rightarrow \mathbb{R}$ be a continuous function such that

$$
\lim _{|x| \rightarrow \infty} \frac{|h(t, x)|}{|x|}=0 \text { uniformly with respect to } a \leq t \leq b \text {. }
$$

Then there exists a continuum $\mathcal{S}$ of positive solutions of the BVPs

$$
\begin{aligned}
& L x-\lambda q(t) x=h(t, x), \\
& x^{(j)}\left(a_{i}\right)=0,1 \leq i \leq m, 0 \leq j \leq k_{i}-1,
\end{aligned}
$$

and $\varepsilon_{o}>0$ such that

1) For each $0<\varepsilon<\varepsilon_{o}$, there is a corresponding subcontinuum $\mathcal{S}_{\varepsilon}$ contained in

$$
U_{\varepsilon}=\left\{(\lambda, u):\left|\lambda-\lambda_{1}(q)\right|<\varepsilon,\|u\|_{P}>\frac{1}{\varepsilon}\right\}
$$

which connects $\left(\lambda_{1}(q), \infty\right)$ and $\partial U_{\varepsilon}$.

2) If $\left(\mu_{l}, x_{l}\right) \in U_{\varepsilon_{0}}$ with $\left\|x_{l}\right\|_{P} \rightarrow \infty$ as $l \rightarrow \infty$, then

$$
\mu_{l} \text { converges to } \lambda_{1}(q) \text { and } \frac{x_{l}}{\left\|x_{l}\right\|_{P}} \text { converges uniformly }
$$

(in fact in $X_{P}$ ) to the unique normalized nontrivial solution of

$$
\left\{\begin{array}{l}
L u=\lambda_{1} q(t) u, \\
u^{(j)}\left(a_{i}\right)=0,1 \leq i \leq m, 0 \leq j \leq k_{i}-1 ;
\end{array}\right.
$$

where $\lambda_{1}:=\lambda_{1}(q)$.

Remark 2.2. An analogue version of Theorem 2.1 can be stated with $q \in \mathbf{L}^{1}((a, b))$ satisfying the following property:

$$
q \neq 0 \quad \text { on a set of positive measure and } S_{P}(t) q(t) \geq 0 \text { for a.e. } t \in(a, b) .
$$

Remark 2.3. It is worth observing that Theorem 2.1 is a generalized version of a result of [1] since this Theorem 2.1 concerns multipoint conjugate boundary conditions and deals with a function $q$ that may vanish on subintervals of $[a, b]$.

For a proof of this Theorem 2.1, we need the lemma below which can also be deduced from Proposition 1.1.

Lemma A. [5]

If $q \in \mathbf{L}^{1}((a, b))$ satisfies $q \neq 0$ on a set of positive measure and $S_{P}(t) q(t) \geq 0$ for a.e. $t \in(a, b)$, then the eigenvalue BVPs

$$
\left\{\begin{array}{l}
L x=\lambda q(t) x, \quad \text { for almost every } t \\
x^{(j)}\left(a_{i}\right)=0,1 \leq i \leq m, 0 \leq j \leq k_{i}-1
\end{array}\right.
$$

has a positive eigenvalue $\lambda_{1}$ which is simple with an eigenfunction $u_{1}$ such that $\inf _{t \neq a_{i}} \frac{u_{1}(t)}{P(t)}>0$. 
Now we recall a standard result on bifurcation theory which together with Lemma A will prove our Theorem 2.1 which is about a bifurcation from infinity for conjugate multipoint BVPs.

Lemma B. [1] [6] [7] [12]

Let $E$ be a real Banach space with norm $\|\cdot\|$. Assume that

$$
T:[a, b] \times E \rightarrow E
$$

is such that for each $\lambda \in[a, b], T(\lambda, \cdot)$ is a compact linear operator, and for each $u \in E, T(\cdot, u)$ is

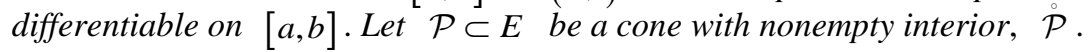

Moreover suppose that

$$
H:[a, b] \times E \rightarrow E
$$

is a completely continuous map satisfying

$$
\lim _{\|u\| \rightarrow \infty} \frac{H(\lambda, u)}{\|u\|}=0 \text { uniformly in } \lambda,
$$

and consider the equation

$$
u=T(\lambda, u)+H(\lambda, u)
$$

If $\left.\lambda_{1} \in\right] a, b\left[, \quad u_{1} \in \stackrel{\circ}{\mathcal{P}}\right.$ with $\left\|u_{1}\right\|=1$,

$$
\operatorname{ker}\left(I-T\left(\lambda_{1}, \cdot\right)\right)=\operatorname{span}\left(u_{1}\right) \text {, and } \frac{\partial T}{\partial \lambda}\left(\lambda_{1}, u_{1}\right) \notin \mathrm{R}\left(I-T\left(\lambda_{1}, \cdot\right)\right) ;
$$

then there exist $\varepsilon_{o}>0$ and a continuum $\mathcal{S} \subset[a, b] \times \mathcal{P}$ such that for any $0<\varepsilon<\varepsilon_{o}$, there exists a corresponding subcontinuum $\mathcal{S}_{\varepsilon} \subset \mathcal{S}$ contained in

$$
U_{\varepsilon}=\left\{(\lambda, u):\left|\lambda-\lambda_{1}\right|<\varepsilon,\|u\|>\frac{1}{\varepsilon}\right\}
$$

which connects $\left(\lambda_{1}, \infty\right)$ and $\partial U_{\varepsilon}$. Moreover if $\left(\mu_{l}, x_{l}\right) \in U_{\varepsilon_{0}}$ with $\left\|x_{l}\right\| \rightarrow \infty$ as $l \rightarrow \infty$; then

$$
\mu_{l} \rightarrow \lambda_{1} \text { and } \frac{x_{l}}{\left\|x_{l}\right\|} \rightarrow u_{1} \text {. }
$$

Proof of Theorem 2.1. First note that all possible solutions of the BVP's $\left(\mathrm{E}_{\lambda}\right)$ lie in $X$ since they are of the form $t \mapsto \int_{a}^{b} G(t, s) f(s) \mathrm{d} s, a \leq t \leq b$; where $f$ is continuous and $G$ is the Green function of the BVPs

$$
\left\{\begin{array}{l}
L x=0, \text { for almost every } t \\
x^{(j)}\left(a_{i}\right)=0,1 \leq i \leq m, 0 \leq j \leq k_{i}-1
\end{array}\right.
$$

with the property that $(t, s) \mapsto \frac{G(t, s)}{P(t)}$ is bounded on $\bigcup_{i=1}^{m-1}\left(a_{i}, a_{i+1}\right) \times[a, b]$.

Now $\left(\mathrm{E}_{\lambda}\right)$ is equivalent, by the properties of the Green function $G$, to the following equation:

$$
u-\lambda T_{q} u=H u ; \quad u \in X .
$$

where $T_{q} u(t)=\int_{a}^{b} G(t, s) q(s) u(s) \mathrm{d} s, a \leq t \leq b$

$$
\text { and } H u(t)=\int_{a}^{b} G(t, s) h(s, u(s)) \mathrm{d} s, a \leq t \leq b .
$$

Moreover as seen in the proof of Lemma A [5], the operator $T_{q}: X \rightarrow X$ is a non-zero positive compact linear operator satisfying

$$
T_{q}(\mathcal{K}) \subset \dot{\mathcal{K}} \cup\{0\},
$$

while $H: X \rightarrow X$ is completely continuous and satisfies

$$
\lim _{\|u\|_{P} \rightarrow \infty} \frac{H u}{\|u\|_{P}}=0
$$


by the assumptions on $h$. Indeed:

1) We show that $H: X \rightarrow X$ is completely continuous.

Step 1. $H$ maps bounded subsets into compact subsets. Let $\left(u_{i}\right)_{i \geq 1}$ be a sequence of elements of $X$ of which norms are bounded, say by a constant real number $C>0$. Let

$$
M_{o}=(b-a) \sup \{|h(\theta, \xi)|: a \leq \theta \leq b,|\xi| \leq C\} .
$$

Then, on one hand,

$$
\left\|u_{i}\right\|_{\infty} \leq C, \forall i
$$

and on the other hand, we have for all $t_{1}, t_{2} \in[a, b]$ :

$$
\begin{aligned}
\left|H u_{i}\left(t_{2}\right)-H u_{i}\left(t_{1}\right)\right| & \leq \int_{a}^{b}\left|G\left(t_{2}, s\right)-G\left(t_{1}, s\right)\right| h\left(s, u_{i}(s)\right) \mid \mathrm{d} s \\
& \leq \frac{M_{o}}{b-a} \int_{a}^{b}\left|G\left(t_{2}, s\right)-G\left(t_{1}, s\right)\right| \mathrm{d} s \\
& \leq M_{o} \sup _{a \leq s \leq b}\left|G\left(t_{2}, s\right)-G\left(t_{1}, s\right)\right| .
\end{aligned}
$$

Hence for all $i$

$$
\left|H u_{i}\left(t_{2}\right)-H u_{i}\left(t_{1}\right)\right| \leq M_{o} \omega_{G}\left(\left|t_{2}-t_{1}\right|\right) ;
$$

where $\omega_{G}$ is the modulus of continuity of $G$. Moreover as a continuous function, $G$ is uniformly continuous on the compact set $[a, b] \times[a, b]$, and so $\lim _{\delta \rightarrow 0^{+}} \omega_{G}(\delta)=0$. Therefore the Ascoli theorem implies the existence of a subsequence $\left(u_{i_{l}}\right)_{l}$ of $\left(u_{i}\right)_{i}$ such that

$$
H u_{i_{l}} \rightarrow v \text { uniformly on }[a, b]
$$

for some $v \in \mathcal{C}([a, b])$.

By applying again Ascoli theorem we see that there exists a subsequence of $\left(u_{i_{l}}\right)_{l}$, still denoted by $\left(u_{i_{l}}\right)_{l}$, such that

$$
\int_{a}^{b} \frac{G(t, s)}{P(t)} h\left(s, u_{i_{l}}(s)\right) \mathrm{d} s \rightarrow w(t)
$$

uniformly on $\bigcup_{i=1}^{m-1}\left(a_{i}, a_{i+1}\right)$ for a suitable $w \in \mathcal{C}([a, b])$. Indeed, to realize this claim, let $\epsilon>0$ and choose $\eta=\eta(\epsilon)>0$ satisfying

$$
\eta<\min \left\{\frac{b-a}{2}, \frac{(b-a) \epsilon}{8 c_{1} M_{o}}\right\}
$$

where $c_{1}$ is a finite upper-bound of the ratio $\frac{G(t, s)}{P(t)}$.

Consider now on the compact $[a, b] \times[a+\eta, b-\eta]$ the function $g_{\eta}$ extending continuously the quotient function $(t, s) \mapsto \frac{G(t, s)}{P(t)}$. The function $g_{\eta}$ is uniformly continuous on $[a, b] \times[a+\eta, b-\eta]$ and so there exists $\delta=\delta(\epsilon)>0$ for which

$$
\omega_{g_{\eta}}(\delta)<\frac{\epsilon}{2\left(M_{o}+1\right)}
$$

Therefore, denoting by $w_{l}$ the continuous extension of $\frac{H u_{i_{l}}}{P}$ to $[a, b]$, i.e.,

$$
w_{l}: x \mapsto \int_{a}^{b} \frac{G(t, s)}{P(t)} h\left(s, u_{i_{l}}(s)\right) \mathrm{d} s
$$


we have for all $t_{1}, t_{2} \in[a, b]$ satisfying $\left|t_{2}-t_{1}\right|<\delta$ :

$$
\begin{aligned}
\left|w_{l}\left(t_{2}\right)-w_{l}\left(t_{1}\right)\right| & \leq \frac{4 c_{1} M_{o}}{b-a} \eta+\frac{M_{o}}{b-a} \int_{a+\eta}^{b-\eta}\left|g_{\eta}\left(t_{2}, s\right)-g_{\eta}\left(t_{1}, s\right)\right| \mathrm{d} s \\
& \leq \frac{\epsilon}{2}+\sup _{a+\eta \leq s \leq b-\eta}\left|g_{h}\left(t_{2}, s\right)-g_{h}\left(t_{1}, s\right)\right| M_{o} \\
& \leq \frac{\epsilon}{2}+\frac{\epsilon}{2} \leq \epsilon, \quad \forall l .
\end{aligned}
$$

This shows that the sequence of functions $\left(w_{l}\right)_{l}$ is equicontinuous on $[a, b]$ and proves the claim since the functions $w_{l}$ are also uniformly bounded as

$$
\left\|w_{l}\right\|_{\infty}=\left\|\frac{H u_{i_{l}}}{P}\right\|_{\infty} \leq M_{o} c_{1} .
$$

Now from the former convergence; i.e. $H u_{i_{l}} \rightarrow v$, we deduce that for all $t \neq a_{1}, \cdots, a_{m}$,

$$
\frac{H u_{i_{l}}(t)}{P(t)} \rightarrow \frac{v(t)}{P(t)}
$$

Thus $w(t)=\frac{v(t)}{P(t)}$ for $t \in \bigcup_{i=1}^{m-1}\left(a_{i}, a_{i+1}\right)$ and it follows that $\left(H u_{i_{l}}\right)_{l}$ converges to $v$ in $X$.

Step 2. If $\left(u_{i}\right)_{i}$ converges to some $u$ in $\left(X,\|\cdot\|_{P}\right) \longmapsto\left(X,\|\cdot\|_{\infty}\right)$, then the Lebesgue dominated convergence theorem implies that $\left(H u_{i}(t)\right)_{i}$ converges to $H u(t)$ for each $t \in[a, b]$.

It follows from the combination of Steps $1 \& 2$ that $H$ is completely continuous; i.e., $H$ maps bounded sets into compact sets and is continuous.

2) We show that $H u=\mathrm{o}\left(\|u\|_{P}\right)$ as $\|u\|_{P} \rightarrow \infty$.

To this end, let $\varepsilon>0$ be arbitrary. Then by assumption there exists $A>0$ such that

$$
|h(t, x)| \leq \frac{\varepsilon}{4(b-a)\left(c_{1}+\|G\|_{\infty}\right)}|x| \text { for all }|x| \geq A \text { and } a \leq t \leq b ;
$$

where $c_{1}>0$ is an upper-bound of $\frac{G(t, s)}{P(t)}$ on $\bigcup_{i=1}^{m-1}\left(a_{i}, a_{i+1}\right) \times[a, b]$. By setting

$M:=\max \{|h(t, x)|: a \leq t \leq b,|x| \leq A\}$, we have at once

$$
|h(t, x)| \leq M+\frac{\varepsilon}{4(b-a)\left(c_{1}+\|G\|_{\infty}\right)}|x| \text { for all } a \leq t \leq b \text { and } x \in \mathbb{R} .
$$

Therefore for every $u \in X$, we have on one hand

$$
|H u(t)| \leq \int_{a}^{b}|G(t, s) h(s, u(s))| \mathrm{d} s \leq M\|G\|_{\infty}(b-a)+\frac{\varepsilon}{4}\|u\|_{\infty}, a \leq t \leq b,
$$

and on the other hand

$$
\left.\left|\frac{H u(t)}{P(t)}\right| \leq M c_{1}(b-a)+\frac{\varepsilon}{4}\|u\|_{\infty}, \quad \forall t \in \bigcup_{i=1}^{m-1}\right] a_{i}, a_{i+1}[.
$$

Thus

$$
\|H u\|_{P} \leq M(b-a)\left(c_{1}+\|G\|_{\infty}\right)+\frac{\varepsilon}{2}\|u\|_{\infty} .
$$

Now by putting

$$
\alpha:=2(b-a)\left(c_{1}+\|G\|_{\infty}\right) \frac{M}{\varepsilon}
$$


we see clearly that

$$
\forall u \in X,\|u\|_{P}>\alpha \Rightarrow \frac{\|H u\|_{P}}{\|u\|_{P}}<\varepsilon
$$

That is

$$
\lim _{\|u\|_{P} \rightarrow \infty} \frac{\|H u\|_{P}}{\|u\|_{P}}=0 .
$$

The result follows by applying Lemma B.

\section{Acknowledgements}

The author is grateful to Professor R. Agarwal for having given him the opportunity to attend the International Conference on the Theory, Methods and Applications of Nonlinear Equations from the $17^{\text {th }}$ to the $21^{\text {st }}$ December 2012.

The author would like also to thank the Abdus-Salam International Centre for Theoretical Physics (ICTP, Trieste, Italy) for its hospitality during his first visit as a Regular Associate.

\section{References}

[1] Coyle, J., Eloe, P.W. and Henderson, J. (1995) Bifurcation from Infinity and Higher Order Ordinary Differential Equations. Journal of Mathematical Analysis and Applications, 195, 32-43. http://dx.doi.org/10.1006/jmaa.1995.1340

[2] Nussbaum, R. (1980) Eigenvectors of Nonlinear Positive Operators and the Linear Krein-Rutman Theorem. Lecture Notes in Mathematics, 886, 309-330.

[3] Schmitt, K. (2005) Positivity, Continuation and Nonlinear Eigenvalue Problems. World Science Publiching, Hackensack, 119-127.

[4] Degla, G. (2001) A Unifying Maximum Principle for Conjugate Boundary Value Problems. Advanced Nonlinear Studies, 1, 121-131.

[5] Degla, G. (2002) On the Principal Eigenvalue of Disconjugate BVPs with L ${ }^{1}$-Coefficients. Advanced Nonlinear Studies, 2, 19-39.

[6] Mawhin, J. (1999) Leray-Schauder Degree: A Half Century of Extensions and Applications. Topological Methods in Nonlinear Analysis. Journal of the Juliusz Schauder Center, 14, 195-228.

[7] Rabinowitz, P. (1983) On Bifurcation from Infinity. Journal of Differential Equations, 14, 462-475. http://dx.doi.org/10.1016/0022-0396(73)90061-2

[8] Mahadevan, R. (2007) A Note on a Nonlinear Krein-Rutman Theorem. Nonlinear Analysis, 67, 3084-3090. http://dx.doi.org/10.1016/j.na.2006.09.062

[9] Chang, K.C. (2009) A Nonlinear Krein-Rutman Theorem. Journal of Systems Science and Complexity, 22, $542-554$. http://dx.doi.org/10.1007/s11424-009-9186-2

[10] Elias, U. (1997) Oscillation Theory of Two-Term Differential Equations. In: Mathematics and Its Applications, Vol. 396, Kluwer Academic Publishers, Dodrecht, 179 p.

[11] Coppel, W.A. (1971) Disconjugacy. In: Lecture Notes in Mathematics, Vol. 220, Springer, New York, 156 p.

[12] Amann, H. (1976) Fixed Point Equations and Nonlinear Eigenvalue Problems in Ordered Banach Spaces. Society for Industrial and Applied Mathematics Review, 18, 620-709. 